Mathematical Research Letters 6, 335-343 (1999)

\title{
CONSTRUCTION OF VALUATIONS FROM $K$-THEORY
}

\author{
IDO EFRAT
}

\begin{abstract}
In this expository paper we describe and simplify results of Arason, Elman, Hwang, Jacob, and Ware on the construction of valuations on a field using $K$-theoretic data.
\end{abstract}

\section{Introduction}

Several recent developments in arithmetic geometry are based on the construction of valuations on a field just from the knowledge of its absolute Galois group. For instance, this is a main ingredient in Pop's proof of the 0-dimensional case of Grothendieck's "anabelian conjecture", saying that any two fields which are finitely generated over $\mathbb{Q}$ and which have isomorphic absolute Galois groups are necessarily isomorphic; see $[\mathrm{P} 2]-[\mathrm{P} 4]$, [S]. Other examples are the characterization of the fields with a $p$-adic absolute Galois group as the $p$-adically closed fields ([E], $[\mathrm{K}]$; see also $[\mathrm{N}],[\mathrm{P} 1])$, and the analogous result for local fields of positive characteristic $[\mathrm{EF}]$.

In the earlier approaches to such results, valuations were detected by means of various local-global principles for Brauer groups (or higher cohomology groups) - often in combination with model-theoretic tools (c.f., [N], [P1]-[P3], [S]). A different approach is introduced in $[\mathrm{E}]$ : there one uses an explicit and elementary construction of valuations which emerged in the mid-1970's in the theory of quadratic forms. It originates from Bröcker's "trivialization of fans" theorem on strictly-pythagorean fields [Br], i.e., real fields $K$ such that $K^{2}+a K^{2} \subseteq K^{2} \cup a K^{2}$ for all $a \in K \backslash\left(-K^{2}\right)$. By Bröcker's result, such a field has a valuation with very special properties: e.g., its value group is non-2-divisible, its residue field is real, and its principal units are squares. An explicit construction of these valuations was given by Jacob $[\mathrm{J}]$ (in the more general context of fans on pythagorean fields). This construction was extended to arbitrary fields by Ware [Wr], and later by Arason, Elman, and Jacob [AEJ]; see [En] for a related result. Roughly speaking, all these results show that if the quadratic forms over the field "behave" as if it possesses a valuation with non-2-divisible value group, residue characteristic $\neq 2$, and such that its principal units are squares, then (apart from a few obvious exceptions) such a valuation actually exists.

Received February 11, 1999.

Mathematics Subject Classification: 12J10 
In the case of an odd prime number $p$ and a field $K$ of characteristic $\neq p$ containing a primitive $p$ th root of unity, Hwang and Jacob [HJ] give an analogous construction of valuations with non- $p$-divisible value group, residue characteristic $\neq p$, and for which the principal units are $p$ th powers. Here the role of quadratic forms is played by certain cohomological structures: the symbolic pairings $K^{\times} / p \otimes_{\mathbb{Z}} K^{\times} / p \rightarrow{ }_{p} \operatorname{Br}(K)$, where ${ }_{p} \operatorname{Br}(K)$ is the $p$-torsion part of the Brauer group of $K$ (see also [Bo] and $[\mathrm{K}]$ for related constructions).

In this expository paper we give a unified and somewhat simplified presentation of these important constructions. Our approach is completely elementary; in particular, we do not use cohomology, nor non-commutative division rings. Further, we do not assume the existence of primitive $p$ th roots of unity in the field. The cohomological structures above are replaced here by the second Milnor $K$-group $K_{2}^{M}(K)$ of $K$, i.e., the quotient of the $\mathbb{Z}$-algebra $K^{\times} \otimes_{\mathbb{Z}} K^{\times}$by the ideal generated by all elements of the form $x \otimes(1-x)$, where $0,1 \neq x \in K$, and the natural projection $K^{\times} \otimes_{\mathbb{Z}} K^{\times} \rightarrow K_{2}^{M}(K), x \otimes y \mapsto\{x, y\}$.

Main Theorem. Let $p$ be a prime number, let $K$ be a field of characteristic $\neq p$, and let $T$ be a subgroup of $K^{\times}$containing $\left(K^{\times}\right)^{p}$ and -1 . Suppose that:

(i) if $x \in K^{\times} \backslash T$ and $y \in T \backslash K^{p}$ then $\{x, y\} \neq 0$;

(ii) if the cosets of $x, y \in K^{\times}$in $K^{\times} / T$ are $\mathbb{F}_{p}$-linearly independent then $\{x, y\} \neq 0$.

Then there exists a valuation ring $O$ on $K$ with value group $\Gamma$, maximal ideal $m$, and residue field $\bar{K}$ such that $(\Gamma: p \Gamma) \geq\left(K^{\times}: T\right) / p, 1-m \subseteq K^{p}$, and char $\bar{K} \neq p$. Furthermore, if $\bar{K}=\bar{K}^{p}$ then $(\Gamma: p \Gamma) \geq\left(K^{\times}: T\right)$.

For a somewhat stronger result see Theorem 4.1.

Needless to say, most ingredients of the proof herein presented already appear in the above-mentioned works. The novelty of this note is mainly in the different organization of the material. We hope that it will make this powerful construction more easily accessible to Galois-theorists. In particular, the construction in this form is already used in $[\mathrm{EF}]$.

I thank Ivan Fesenko for the encouragement to publish this manuscript, and for his helpful remarks.

\section{The sets $O^{+}, O^{-}$}

From now on we fix a field $K$ and a subgroup $T$ of $K^{\times}$. Let

$$
A=\left\{x \in K^{\times} \mid T-x T \nsubseteq T \cup-x T\right\},
$$

and let $B=\langle-1, A\rangle$ be the subgroup of $K^{\times}$generated by -1 and $A$.

\section{Remark 1.1.}

(i) If $x \in T$ then $0 \in T-x T$ while $0 \notin T \cup-x T$. Thus $T \subseteq A$.

(ii) For $x \in K^{\times}$one has $x \in A$ if and only if $x^{-1} \in A$.

(iii) If $x \in K^{\times} \backslash T$ and $1-x \notin T \cup-x T$ then $1-x \in A$ : indeed, $x \in T-(1-x) T$ but $x \notin T \cup-(1-x) T$. 
Given a subgroup $S$ of $K^{\times}$we denote $O^{-}(S)=(1-T) \backslash S$.

Lemma 1.2. If $z, w \in O^{-}(B)$ then either $z w \in 1-T$ or $1-z w \in z T=w T$.

Proof. One has $-z,-w \notin B$, so

$$
\begin{aligned}
& 1-z w=(1-z)+z(1-w) \in T+z T \subseteq T \cup z T \\
& 1-z w=(1-w)+w(1-z) \in T+w T \subseteq T \cup w T .
\end{aligned}
$$

Proposition 1.3. Suppose that there exist $a, b \in O^{-}(B)$ with $1-a b \notin T$. Then:

(a) $O^{-}(\langle B, a\rangle) O^{-}(\langle B, a\rangle) \subseteq 1-T$;

(b) $A=T$;

(c) $T-a^{2} T \nsubseteq T \cup a^{2} T$.

Proof. (a) Let $H=\langle B, a\rangle$. Lemma 1.2 implies that $1-a b \in a T=b T$, whence $b \in H$. Suppose that $0 \neq x, y \in O^{-}(H)$ but $x y \notin 1-T$. As $a, b \in H, x, y \notin H$, and $T \leq H$, Lemma 1.2 implies that $a x, b y \in 1-T$. Furthermore, $a x, b y \notin H$, so $a x, b y \in O^{-}(B)$. As $a y^{-1} \notin H$, also $a y^{-1} \notin A$. Hence one of the following cases holds:

CAse (I): $a y^{-1} \in 1-T$. Then $a y^{-1} \in O^{-}(B)$ and $\left(a y^{-1}\right)(b y)=a b \notin 1-T$. By Lemma 1.2, $1-a b \in a y^{-1} T$, contrary to $1-a b \in a T$ and $y \notin H$.

CASE (II): $\quad a^{-1} y \in 1-T$. Then $a^{-1} y \in O^{-}(B)$ and $x y=(a x)\left(a^{-1} y\right) \notin 1-T$. By applying Lemma 1.2 twice we obtain $1-x y \in x T \cap a x T$, contrary to $a \notin B$.

(b) By Remark 1.1 (i), $T \subseteq A$. Conversely, take $x \in A$. Suppose $x \notin T$. After replacing $x$ by an appropriate element of $x T$, we may assume that $1-x \notin$ $T \cup-x T$.

By Remark 1.1 (iii), $1-x \in A \subseteq B$. Since $x \in B$ and $a \notin B$ we have $x a,-(1-x) a \notin B$. In particular, $x a,-(1-x) a \notin A$. Therefore

$$
\begin{aligned}
& 1-x a \in T-x a T \subseteq T \cup-x a T \\
& 1-x a \in T+(1-x) a T \subseteq T \cup(1-x) a T .
\end{aligned}
$$

By the choice of $x$, the cosets $-x a T$ and $(1-x) a T$ are disjoint. Hence $1-x a \in T$, so $x a \in O^{-}(B)$.

Since also $x^{-1} \in A$ (Remark 1.1 (ii)) and since $1-x^{-1} \notin T \cup-x^{-1} T$, the same argument (with $x, a$ replaced by $x^{-1}, b$ ) shows that $x^{-1} b \in O^{-}(B)$. As $a b=(x a)\left(x^{-1} b\right) \notin 1-T$, Lemma 1.2 implies that $1-a b \in a T \cap x a T$. This contradicts $x \notin T$.

(c) As already noted, $1-a b \in a T=b T$ and $a \notin T$. Hence $1-a b \in T-a^{2} T$ but $1-a b \notin T \cup a^{2} T$.

Next we define a group $H$ as follows:

- If $O^{-}(B) O^{-}(B) \subseteq 1-T$ then we take $H=B$;

- If $O^{-}(B) O^{-}(B) \nsubseteq 1-T$ then we choose $a \in O^{-}(B)$ such that $a O^{-}(B) \nsubseteq$ $1-T$ and set $H=\langle B, a\rangle$. 
Thus $\pm T \leq \pm A \leq B \leq H$. We abbreviate $O^{-}=O^{-}(H)$, and let

$$
O^{+}=\left\{x \in H \mid x O^{-} \subseteq O^{-}\right\}
$$

\section{Proposition 1.4.}

(a) $\mathrm{O}^{-} \mathrm{O}^{-} \subseteq 1-\mathrm{T}$.

(b) $1-O^{-} \subseteq O^{+}$.

(c) $O^{-} O^{-} \subseteq 1-O^{+}$.

(d) $\left(1-O^{+}\right) \cap H \subseteq O^{+}$.

(e) $\left(1-O^{+}\right) \backslash H \subseteq O^{-}$.

Proof. (a) follows from Proposition 1.3 (a). For $1 \neq y \in K$ let $\tilde{y}=y /(y-1)$. Then $y \mapsto \tilde{y}$ maps $K \backslash\{0,1\}$ onto itself. Moreover, $y \in O^{-}$if and only if $\tilde{y} \in O^{-}$. We use the identity

$$
1-x y=(1-(1-x) \tilde{y})(1-y)
$$

for $y \neq 1$.

(b) Take $x \in 1-O^{-}$and $y \in O^{-}$. By (*) and (a), $1-x y \in\left(1-O^{-} O^{-}\right) T \subseteq$ $T T=T$. Since $x \in T \leq H$ and $y \notin H$ this implies $x y \in O^{-}$. Conclude that $x \in O^{+}$.

(c) Let $x, y \in O^{-}$. By $(*)$ and (b),

$$
\begin{aligned}
1-x y \in\left(1-\left(1-O^{-}\right) O^{-}\right)\left(1-O^{-}\right) \subseteq\left(1-O^{+} O^{-}\right) O^{+} \\
\\
\quad \subseteq\left(1-O^{-}\right) O^{+} \subseteq O^{+} O^{+} \subseteq O^{+} .
\end{aligned}
$$

(d) Suppose that $x \in\left(1-O^{+}\right) \cap H$ and $y \in O^{-}$. By (*),

$$
1-x y \in\left(1-O^{+} O^{-}\right)\left(1-O^{-}\right) \subseteq\left(1-O^{-}\right)\left(1-O^{-}\right) \subseteq T T=T .
$$

As $x y \notin H$, this shows that $x y \in O^{-}$, whence $x \in O^{+}$.

(e) If $x \in\left(1-O^{+}\right) \backslash H$ then $x \notin A$, so $1-x \in H \cap(T \cup-x T)=T$. Conlcude that $x \in O^{-}$.

\section{The valuation $O$}

Let $A, H, O^{-}, O^{+}$be as in $\S 1$, and let $O=O^{-} \cup O^{+}$.

Proposition 2.1. $O$ is a valuation ring on $K$.

Proof. We apply (a)-(e) of Proposition 1.4.

By definition, $O^{+} O^{-} \subseteq O^{-}$and $O^{+} O^{+} \subseteq O^{+}$. As $O^{-} O^{-} \subseteq 1-T$ also $O^{-} O^{-} \backslash H \subseteq O^{-}$. Finally, $O^{-} O^{-} \cap H \subseteq\left(1-O^{+}\right) \cap H \subseteq O^{+}$. Conclude that $O O \subseteq O$. 
Next we show that for every $0 \neq x \in K$ either $x \in O$ or $x^{-1} \in O$. Indeed, if $x \notin H$ then $x \notin A$, so either $1-x \in T$ or $1-x^{-1} \in T$. Thus either $x \in O^{-}$or $x^{-1} \in O^{-}$in this case. If $x \in H \backslash \in O^{+}$then there exists $y \in O^{-}$ such that $x y \notin O^{-}$. By what we have just seen, $(x y)^{-1} \in O^{-}$. Consequently, $x^{-1}=(x y)^{-1} y \in O^{-} O^{-} \subseteq O O \subseteq O$, as desired. In particular, $\pm 1 \in O$.

As $1-O^{-} \subseteq O^{+},\left(1-O^{+}\right) \cap H \subseteq O^{+}$, and $\left(1-O^{+}\right) \backslash H \subseteq O^{-}$, we have $1-O \subseteq O$.

For $0 \neq x, y \in O$ we show that $x+y \in O$. By symmetry we may assume that $-x^{-1} y \in O$. Then $1+x^{-1} y \in 1-O \subseteq O$. Therefore $x+y=x\left(1+x^{-1} y\right) \in$ $O O \subseteq O$.

The assertion follows.

Proposition 2.2. $O^{\times} \leq H$.

Proof. Otherwise there exists $x \in O^{\times} \backslash H$. In particular, $x \in O^{-}$, so $1-x \in T$. Hence $1-x^{-1} \in-x^{-1} T$, and therefore $1-x^{-1} \notin T$. Conclude that $x^{-1} \notin O^{-}$, contrary to $x \in O^{\times} \backslash H$.

We denote the maximal ideal of the valuation $O$ by $m$.

Proposition 2.3. $1-m \leq T$.

Proof. By definition, $1-O^{-} \subseteq T$. So let $x \in O^{+} \cap m$; we show that $x \in 1-T$. As $x^{-1} \in H \backslash O^{+}$we have $x^{-1} y \notin O^{-}$for some $y \in O^{-}$. Since $x^{-1} y \notin H$ this implies $x^{-1} y \notin O$. Hence $x y^{-1} \in O \backslash H=O^{-}$. By Proposition 1.4 (a), $x=\left(x y^{-1}\right) y \in O^{-} O^{-} \subseteq 1-T$.

Fix a prime number $p$.

Lemma 2.4. If $1-(m \backslash H) \subseteq\left(K^{\times}\right)^{p}$ then $1-m \subseteq\left(K^{\times}\right)^{p}$.

Proof. Take $m \in m \cap H$. Since $m^{-1} \notin O^{+}$there exists $y \in O^{-}$such that $m^{-1} y \notin$ $O^{-}$. As $m^{-1} y \notin H$ this means that $m^{-1} y \notin O$. Then $y, y^{-1} m \in O \backslash H \subseteq m$, by Proposition 2.2. By Proposition 2.3, $1+y^{-1} m-m \in 1-m \leq T \leq H$. Since $y \in m \backslash H$ this implies $y+m-y m \in m \backslash H$. By assumption, $(1-y)(1-m)=$ $1-(y+m-y m) \in\left(K^{\times}\right)^{p}$. Also, $1-y \in 1-(m \backslash H) \subseteq\left(K^{\times}\right)^{p}$. Hence $1-m \in\left(K^{\times}\right)^{p}$.

Corollary 2.5. Suppose that for every $x \in K^{\times} \backslash H$ and every $y \in T \backslash\left(K^{\times}\right)^{p}$ one has $\{x, y\} \neq 0$. Then $1-m \subseteq\left(K^{\times}\right)^{p}$.

Proof. Let $x \in m \backslash H$. Then $x \in O^{-}$, so $1-x \in T$. As $\{x, 1-x\}=0$ we have $1-x \in\left(K^{\times}\right)^{p}$. Now apply Lemma 2.4 .

Lemma 2.6. Suppose that $p \in m$ and $1-m \subseteq\left(K^{\times}\right)^{p}$. Then $m \backslash p m \subseteq\left(K^{\times}\right)^{p}$.

Proof. Given $x \in 1-m$, we may write $x=y^{p}$ with $y \in O^{\times}$. The residues $\bar{x}, \bar{y}$ then satisfy $\overline{1}=\bar{x}=\bar{y}^{p}$. Since char $O / m=p$, necessarily $\bar{y}=\overline{1}$, i.e., $y \in 1-m$. Thus $1-m=(1-m)^{p}$. 
Now let $a \in m \backslash p m$. By what we have just seen, there exists $b \in m$ such that $1+a=(1+b)^{p} \in 1+b^{p}-p m$. Since $a \notin p m$ this implies $a \in b^{p}(1-m) \subseteq\left(K^{\times}\right)^{p}$.

From now on we assume that $\left(K^{\times}\right)^{p} \leq T$.

Corollary 2.7. If $1-m \subseteq\left(K^{\times}\right)^{p}$ and char $K \neq p$ then $p \notin m$.

Proof. Suppose $p \in m$. Lemma 2.6 then shows that $p \in m \backslash p m \subseteq\left(K^{\times}\right)^{p} \leq H$. Since $p^{-1} \notin O^{+}$, there exists $a \in O^{-}$such that $p^{-1} a \notin O$. By Proposition 2.2, $O^{\times} \leq H$, so $a \in m \backslash p m$. Lemma 2.6 once again gives $a \in\left(K^{\times}\right)^{p} \leq H$, a contradiction.

\section{The size of $H$}

In order to prove the non-triviality of $O$ in various situations one needs an estimate on the size of $(H: T)$. This is obtained in Corollary 3.3 below. For its proof we need two technical facts.

Lemma 3.1. Let $\Delta$ be an elementary abelian $p$-group and let $\omega: \Delta \rightarrow \mathbb{Z} / p$ be a map such that:

(i) if $a, b \in \Delta$ are $\mathbb{F}_{p}$-linearly independent and at least one of $\omega(a), \omega(b)$ is non-zero then $\omega(a b)=\omega(a) \omega(b)$;

(ii) there exist $\mathbb{F}_{p}$-linearly independent $a, b \in \Delta$ such that $\omega(a), \omega(b) \neq 0$.

Then $1 \in \operatorname{Im}(\omega)$.

Proof. Take $a, b$ as in (ii). From (i) we obtain inductively that

$$
\omega\left(a^{i} b\right)=\omega(a)^{i} \omega(b) \neq 0,
$$

$i=1, \ldots, p-1$. Since $(\mathbb{Z} / p)^{\times}$has order $p-1$ this gives in particular $\omega\left(a^{p-1} b\right)=$ $\omega(b)$. Moreover, $\omega\left(a^{p-1}\right) \omega(b)=\omega\left(a^{p-1} b\right)$ by (i). Hence $\omega\left(a^{p-1}\right)=1$.

Proposition 3.2. Assume that for every $x \in K^{\times} \backslash T$ one has $1-x \in \bigcup_{i=0}^{p-1} x^{i} T$. Suppose that the cosets of $a, b \in K^{\times}$in $K^{\times} / T$ are $\mathbb{F}_{p}$-linearly independent. Then $1-a \in T \cup a T$ or $1-b \in T \cup b T$.

Proof. For every $x \in K^{\times} \backslash T$ there exists by assumption a unique $0 \leq i \leq p-1$ such that $1-x \in x^{i} T$. When $i \neq 0$ let $0 \leq \omega(x) \leq p-1$ be the unique integer such that $w(x) \equiv 1-i^{-1}(\bmod p)$. When $i=0$ we set $\omega(x)=0$. Note that $\omega(x)=0$ if and only if $1-x \in T \cup x T$. Also, $1 \notin \operatorname{Im}(\omega)$.

We apply Lemma 3.1 with $\Delta=K^{\times} / T$. It suffices to show that if the cosets of $a, b \in K^{\times}$in $K^{\times} / T$ are $\mathbb{F}_{p}$-linearly independent and at least one of $\omega(a), \omega(b)$ is non-zero then $\omega(a b) \equiv \omega(a) \omega(b)(\bmod p)$.

Take $0 \leq i, j, r \leq p-1$ such that $1-a \in a^{i} T, 1-b \in b^{j} T, 1-a b \in(a b)^{r} T$. The assumptions imply that $i \neq 1$ or $j \neq 0$. Hence

$$
1-a b=(1-a)+a(1-b) \in a^{i}\left(T-a^{1-i} b^{j} T\right) \subseteq \bigcup_{k=0}^{p-1} a^{i}\left(a^{1-i} b^{j}\right)^{k} T .
$$


Therefore, $(a b)^{r} T \cap a^{i}\left(a^{1-i} b^{j}\right)^{k} T \neq \emptyset$ for some $0 \leq k \leq p-1$. Since $a, b$ are independent modulo $T$ one has $r \equiv i+(1-i) k \equiv j k(\bmod p)$. Then $r(i+j-1) \equiv j k(i+j-1) \equiv i j(\bmod p)$.

If $r \neq 0$ then also $i, j \neq 0$ and $1-r^{-1} \equiv\left(1-i^{-1}\right)\left(1-j^{-1}\right)(\bmod p)$; i.e., $\omega(a b) \equiv \omega(a) \omega(b)(\bmod p)$, as required.

If $r=0$ then either $i=0$ or $j=0$, so either $\omega(a b)=\omega(a)=0$ or $\omega(a b)=$ $\omega(b)=0$, and we are done again.

Corollary 3.3. Suppose that $-1 \in T$ and that for every $x \in K^{\times} \backslash T$ one has $1-x \in \bigcup_{i=0}^{p-1} x^{i} T$. Then $(H: T) \mid p$.

Proof. By Proposition 3.2, $(B: T) \mid p$. Now if $O^{-}(B) O^{-}(B) \subseteq 1-T$ then $H=B$, so $(H: T) \mid p$. If $O^{-}(B) O^{-}(B) \nsubseteq 1-T$ then $A=T$, by Proposition 2.2(b); hence $B=T$, so $(H: T)=(H: B)=p$.

\section{The main result}

By combining the previous results we now obtain:

Theorem 4.1. Let $K$ be a field and let $\left(K^{\times}\right)^{p} \leq T \leq K^{\times}$be an intermediate group. Suppose that:

(i) if $x \in K^{\times} \backslash T$ and $y \in T \backslash K^{p}$ then $\{x, y\} \neq 0$;

(ii) if $-1 \in T$ and if the cosets of $x, y \in K^{\times}$in $K^{\times} / T$ are $\mathbb{F}_{p}$-linearly independent then $\{x, y\} \neq 0$.

Then $O$ above is a valuation ring. Furthermore, let $m, \bar{K}$, and $\Gamma$, be its maximal ideal, residue field, and value group, respectively. Then:

(a) $1-m \subseteq\left(K^{\times}\right)^{p}$;

(b) if char $K \neq p$ then also char $\bar{K} \neq p$;

(c) if $-1 \in T$ then $\left(O^{\times} T: T\right) \leq p$;

(d) if $-1 \notin T$ then $\left(O^{\times} B: B\right) \leq 2$;

(e) if $-1 \in T$ then $(\Gamma: p \Gamma) \geq\left(K^{\times}: T\right) / p$;

(f) if $-1 \notin T$ then $(\Gamma: 2 \Gamma) \geq\left(K^{\times}: B\right) / 2$;

(g) if $\bar{K}=\bar{K}^{p}$ and $-1 \in T$ then $(\Gamma: p \Gamma) \geq\left(K^{\times}: T\right)$;

(h) if $\bar{K}=\bar{K}^{p}$ and $-1 \notin T$ then $(\Gamma: 2 \Gamma) \geq\left(K^{\times}: B\right)$.

Proof. By Proposition 2.1, $O$ is a valuation ring. Assumption (i) and Corollary 2.5 prove (a). Corollary 2.7 proves (b). By Proposition 2.2, $O^{\times} \subseteq H$.

Suppose that $-1 \in T$. For every $x \in K^{\times} \backslash T$ one has $\{x, 1-x\}=0$, so by (ii), $1-x \in \bigcup_{i=0}^{p-1} x^{i} T$. Corollary 3.3 now gives $(H: T) \mid p$, whence (c). Furthermore,

$$
(\Gamma: p \Gamma)=\left(K^{\times}: O^{\times}\left(K^{\times}\right)^{p}\right) \geq\left(K^{\times}: H\right) \geq\left(K^{\times}: T\right) / p,
$$

proving (e).

To prove (g), suppose that $\bar{K}=\bar{K}^{p}$. By Lemma $2.3, O^{\times}=(1-m)\left(O^{\times}\right)^{p} \leq T$. If $H=O^{\times}\left(K^{\times}\right)^{p}$ then $H \leq T$; hence $H=T$, so $(\Gamma: p \Gamma)=\left(K^{\times}: T\right)$, and we 
are done in this case. On the other hand, if $H>O^{\times}\left(K^{\times}\right)^{p}$ then the inequalities above show that $(\Gamma: p \Gamma)>\left(K^{\times}: T\right) / p$. Thus (g) holds in this case as well.

When $-1 \notin T$ we have $p=2$ and $(H: B) \leq 2$. Assertions (d),(f), and (h) are then proven similarly to (c), (e), and (g).

Remark 4.2. If $p=2$ and $-1 \in T$ then assumption (ii) of Theorem 4.1 implies that for every $x \in K^{\times} \backslash T$ one has $1-x \in T \cup x T$. Hence $T=A=B$. This shows that the Main Theorem as stated in the introduction is a special case of Theorem 4.1.

Example 4.3. Let $p$ be a prime number and let $K$ be a field. Suppose that the canonical symbolic map induces an isomorphism $\wedge^{2}\left(K^{\times} / p\right) \cong K_{2}^{M}(K) / p$. Then (i) and (ii) of Theorem 4.1 hold with $T=\left(K^{\times}\right)^{p}$. Hence $K$ possesses a valuation satisfying (a)-(g) above.

In particular, this happens for $K=\mathbb{F}_{l}\left(\left(t_{1}\right)\right) \cdots\left(\left(t_{n}\right)\right)$, where $l$ is a prime number such that $p \mid l-1$ and such that $4 \mid l-1$ if $p=2[\mathrm{Wd}, \S 2]$. Then $\mathbb{F}_{l}$ contains a primitive $p$ th root of unity, and $\left(K^{\times}:\left(K^{\times}\right)^{p}\right)=p^{n+1}[\mathrm{Wd}$, Lemma 1.4]. Moreover, the value group $\Gamma$ of every valuation on $K$ satisfies $(\Gamma: p \Gamma) \leq$ $p^{n}$. This shows that condition (e) of Theorem 4.1 cannot be strengthened to $(\Gamma: p \Gamma) \geq\left(K^{\times}: T\right)$.

We conclude by proving a criterion for the existence of valuations having arbitrary residue characteristic:

Theorem 4.4. Let $p$ be an odd prime and let $K$ be a field. The following conditions are equivalent:

(a) There exists a valuation $v$ on $K$ with non-p-divisible value group;

(b) There exists an intermediate group $\left(K^{\times}\right)^{p} \leq T<K^{\times}$such that for every $x \in K^{\times} \backslash T$ one has $1-x \in T \cup x T$.

Proof. $(\mathrm{a}) \Rightarrow(\mathrm{b})$ : Let $T=v^{-1}(p \Gamma)$ and take $x \in K^{\times}$. When $v(x)=0$ (resp., $v(x)>0, v(x)<0$ ) we have $x \in T$ (resp., $1-x \in T, 1-x \in x T$ ).

(b) $\Rightarrow(\mathrm{a})$ : We take $T$ as in (b). Since $p \neq 2$ we have $-1 \in T$, so $B=A=T$. Moreover, if $a \notin T$ then $a^{2} \notin T$, so $T-a^{2} T \subseteq T \cup a^{2} T$. By Proposition 1.3(c), $O^{-}(T) O^{-}(T) \subseteq 1-T$, whence $H=T$. Propositions 2.1 and 2.2 give rise to a valuation ring $O$ such that $O^{\times} \leq T$. Its value group $\Gamma$ satisfies $(\Gamma: p \Gamma)=\left(K^{\times}\right.$: $\left.O^{\times}\left(K^{\times}\right)^{p}\right) \geq\left(K^{\times}: T\right)>1$.

\section{References}

[AEJ] J.K. Arason, R. Elman and B. Jacob, Rigid elements, valuations, and realization of Witt rings, J. Algebra 110 (1987), 449-467.

[Bo] F.A. Bogomolov, Abelian subgroups of Galois groups, Izv. Akad. Nauk SSSR Ser. Mat. 55 (1991), 32-67 (Russian); translation in Math. USSR Izv. 38 (1992), 27-67.

[Br] L. Bröcker, Characterization of fans and hereditarily pythagorean fields, Math. Z. 151 (1976), 149-163.

[E] I. Efrat, A Galois-theoretic characterization of p-adically closed fields, Isr. J. Math. 91 (1995), 273-284. 
[EF] I. Efrat and I. Fesenko, Fields Galois-equivalent to a local field of positive characteristic, Math. Res. Lett., to appear.

[En] A.J. Engler, Totally real rigid elements and $F_{\pi}$-henselian valuation rings, Comm. Algebra 25 (1997), 3673-3697.

[HJ] Y.S. Hwang and B. Jacob, Brauer group analogues of results relating the Witt ring to valuations and Galois theory, Canad. J. Math. 47 (1995), 527-543.

[J] B. Jacob, On the structure of pythagorean fields, J. Algebra 68 (1981), 247-267.

[K] J. Koenigsmann, From p-rigid elements to valuations (with a Galois-characterisation of $p$-adic fields), With an appendix by F. Pop, J. Reine Angew. Math. 465 (1995), $165-182$.

[N] J. Neukirch, Kennzeichnung der p-adischen und endlichen algebraischen Zahlkörper, Invent. Math. 6 (1969), 269-314.

[P1] F. Pop, Galoissche Kennzeichnung p-adisch abgeschlossener Körper, J. Reine Angew. Math. 392 (1988), 145-175.

[P2] - On Grothendieck's conjecture of birational anabelian geometry, Ann. Math. 139 (1994), 145-182.

[P3] - On Grothendieck's conjecture of birational anabelian geometry II, preprint, Heidelberg 1995.

[P4] Glimpses of Grothedieck's anabelian geometry, Geometric Galois actions. 1 (L. Schneps et al., ed.), Lond. Math. Soc. Lect. Note Ser., vol. 242, Cambridge University Press, Cambridge, 1997, pp. 113-126.

[S] M. Spiess, An arithmetic proof of Pop's Theorem concerning Galois groups of function fields over number fields, J. Reine Angew. Math. 478 (1996), 107-126.

[Wd] A.R. Wadsworth, p-henselian fields: K-theory, Galois cohomology, and graded Witt rings, Pac. J. Math. 105 (1983), 473-496.

[Wr] R. Ware, Valuation rings and rigid elements in fields, Canad. J. Math. 33 (1981), $1338-1355$.

Department of Mathematics and Computer Science, Ben Gurion University of the Negev, P.O. Box 653, BE'ER-Sheva 84105, ISRAEL

E-mail address: efrat@math.bgu.ac.il 